\title{
Impact of a cometary outburst on its ionosphere
}

\section{Rosetta Plasma Consortium observations of the outburst exhibited by comet 67P/Churyumov-Gerasimenko on 19 February 2016}

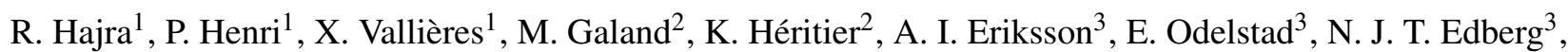 \\ J. L. Burch ${ }^{4}$, T. Broiles ${ }^{4}$, R. Goldstein ${ }^{4}$, K. H. Glassmeier ${ }^{5}$, I. Richter ${ }^{5}$, C. Goetz ${ }^{5}$, B. T. Tsurutani ${ }^{6}$, H. Nilsson ${ }^{7}$, \\ K. Altwegg ${ }^{8}$, and M. Rubin ${ }^{8}$ \\ ${ }^{1}$ Laboratoire de Physique et Chimie de l'Environnement et de l'Espace (LPC2E), CNRS, Orléans, France \\ e-mail: rajkumar.hajra@cnrs-orleans.fr, rajkumarhajra@yahoo.co.in \\ 2 Imperial College, South Kensington Campus, London SW7 2AZ, UK \\ 3 Institutet för rymdfysik, Ångström Laboratory, Lagerhyddsvagen 1, 75105 Uppsala, Sweden \\ ${ }^{4}$ Southwest Research Institute, PO Drawer 28510, San Antonio, TX 78228-0510, USA \\ 5 Institut für Geophysik und extraterrestrische Physik, TU Braunschweig, Mendelssohnstr. 3, 38106 Braunschweig, Germany \\ 6 Jet Propulsion Laboratory, California Institute of Technology, 4800 Oak Grove Drive, Pasadena, CA 91109, USA \\ 7 Swedish Institute of Space Physics, PO Box 812, 98128 Kiruna, Sweden \\ 8 Physikalisches Institut, Universität Bern, Sidlerstr. 5, 3012 Bern, Switzerland
}

Received 10 February 2017 / Accepted 27 June 2017

\begin{abstract}
We present a detailed study of the cometary ionospheric response to a cometary brightness outburst using in situ measurements for the first time. The comet 67P/Churyumov-Gerasimenko (67P) at a heliocentric distance of $2.4 \mathrm{AU}$ from the Sun, exhibited an outburst at $\sim 1000$ UT on 19 February 2016, characterized by an increase in the coma surface brightness of two orders of magnitude. The Rosetta spacecraft monitored the plasma environment of 67P from a distance of $30 \mathrm{~km}$, orbiting with a relative speed of $\sim 0.2 \mathrm{~m} \mathrm{~s}^{-1}$. The onset of the outburst was preceded by pre-outburst decreases in neutral gas density at Rosetta, in local plasma density, and in negative spacecraft potential at $\sim 0950$ UT. In response to the outburst, the neutral density increased by a factor of $\sim 1.8$ and the local plasma density increased by a factor of $\sim 3$, driving the spacecraft potential more negative. The energetic electrons (tens of eV) exhibited decreases in the flux of factors of $\sim 2$ to 9 , depending on the energy of the electrons. The local magnetic field exhibited a slight increase in amplitude $(\sim 5 \mathrm{nT})$ and an abrupt rotation $\left(\sim 36.4^{\circ}\right)$ in response to the outburst. A weakening of $10-100 \mathrm{mHz}$ magnetic field fluctuations was also noted during the outburst, suggesting alteration of the origin of the wave activity by the outburst. The plasma and magnetic field effects lasted for about $4 \mathrm{~h}$, from 1000 UT to $1400 \mathrm{UT}$. The plasma densities are compared with an ionospheric model. This shows that while photoionization is the main source of electrons, electron-impact ionization and a reduction in the ion outflow velocity need to be accounted for in order to explain the plasma density enhancement near the outburst peak.
\end{abstract}

Key words. plasmas - waves - methods: data analysis - methods: observational - comets: general comets: individual: 67P/Churyumov-Gerasimenko

\section{Introduction}

The interaction of an active comet with the solar wind is an interesting physical process in the context of mass-loaded space plasmas (e.g., Szegö et al. 2000; Coates \& Jones 2009; Glassmeier 2017). Previous studies concentrate on interaction scenarios where the activity is steady. A very different interaction situation is expected for cometary outbursts. Cometary outbursts are the most spectacular aspect of cometary activity. They are defined by an abrupt increase in cometary brightness followed by a gradual decrease to the pre-event brightness. In general, during an outburst, the comet brightness increases by a factor of $\sim 2-5$ within a few hours. This corresponds to an average mass release of $\sim 100 \mathrm{~kg}$ with speed in the range of $\sim 0.1-1.0 \mathrm{~km} \mathrm{~s}^{-1}$ and an average kinetic energy release of $\sim 10^{12 \pm 4} \mathrm{~J}$ (Whitney 1955; Hughes 1990; Beech \& Gauer 2002; Gronkowski \& Wesolowski 2015). The largest outburst was detected at comet 17P/Holmes on
24 October 2007, which had a brightness increase of 15 mag (Moreno et al. 2008; Sekanina 2008a).

Outbursts are reported to occur independently of the heliocentric distance from the Sun (West et al. 1991; Filonenko \& Churyumov 2006; Sekanina 2008b; Belton et al. 2013). The proposed energy sources for the outbursts can be classified into three groups: (1) internal sources related to the energy stored in the comet nucleus, such as the crystallization of amorphous water ice, polymerization of hydrogen cyanide $(\mathrm{HCN})$, and chemical reactions (Rettig et al. 1992; Gronkowski 2007; Gronkowski \& Sacharczuk 2010; Qi et al. 2015; Miles 2016); (2) external sources, such as collision of comets with meteoroids and asteroids, the influence of solar flares and solar wind (Huebner \& Weigert 1966; Niedner 1980; Intrilligator \& Dreyer 1991; Ibadov 2012); and (3) changes in the internal structure and strength of the cometary material (Tambovtseva \& Shestakova 1999; Gronkowski 2009). If 
comets break up into pieces, large areas of fresh snow and ice are exposed to the influence of the solar radiation resulting in cometary outbursts.

Although cometary brightness outbursts have been studied for more than $100 \mathrm{yr}$ (Eddington 1910; Hughes 1990; Miles 2016; Vincent et al. 2016, and references therein), our present understanding is mainly based on remote-sensing and fly-by observations and on modeling. The Rosetta orbiter (Glassmeier et al. 2007a) of the European Space Agency (ESA) observed an outburst at comet $67 \mathrm{P} /$ Churyumov-Gerasimenko (hereafter 67P) on 19 February 2016 (Grün et al. 2016). It was the first reported in situ observation of a cometary outburst. Images taken by the Wide Angle Camera (WAC) of the Optical, Spectroscopic, and Infrared Remote Imaging System (OSIRIS; Keller et al. 2007) on board Rosetta showed an increase of two orders of magnitude in the 67P coma surface brightness between 0940 and 1010 UT. An overview of the multi-instrument observations of the outburst can be found in Grün et al. (2016). The outburst originated from the Atum region of the comet (at latitude $-28.6^{\circ}$ and longitude $232.3^{\circ}$ ), and was thought to be triggered by thermal stress in the surface material together with fracture mechanics and gravity in the form of landslides that exposed water ice to direct solar illumination.

The purpose of the present paper is to explore in detail, for the first time, the cometary plasma response to an outburst through in situ observations. The plasma and magnetic field responses of the cometary outburst are characterized.

This article is organized as follows. The plasma, particle, and magnetic field observations made by the Rosetta Plasma Consortium (RPC; Carr et al. 2007) sensors on board the Rosetta spacecraft along with the neutral gas observations from the Rosetta Orbiter Spectrometer for Ion and Neutral Analysis (ROSINA; Balsiger et al. 2007) are presented in Sect. 2.1. Further discussion on the particle and field data are given in Sects. 2.2-2.4. Observed plasma densities are compared with an ionospheric model in Sect. 3.1, and the variation of magnetic wave activity is discussed in Sect. 3.2. The results are summarized and the implications of the present in situ observations for further remote-sensing studies are discussed in Sect. 4.

\section{Observations}

The impact of the comet 67P outburst on 19 February 2016 on the cometary ionosphere and induced magnetosphere is studied using in situ measurements by the RPC on board the Rosetta orbiter spacecraft. The neutral gas densities measured by the COmet Pressure Sensor (COPS) from the ROSINA are also used in the present study. The RPC is a suite of five plasma instruments for complementary measurements of the plasma environment around comet 67P. It is composed of a Mutual Impedance Probe (MIP; Trotignon et al. 2007), two LAngmuir Probes (LAP; Eriksson et al. 2007), an Ion and Electron Sensor (IES; Burch et al. 2007), an Ion Composition Analyzer (ICA; Nilsson et al. 2007), and two fluxgate MAGnetometers (MAG; Glassmeier et al. 2007b).

\subsection{Ionospheric response to the cometary outburst on 19 February 2016: an overview}

Figure 1 shows the available RPC measurements of the cometary plasma and magnetic field along with the neutral gas density measured by ROSINA/COPS from 0800 UT to 1600 UT on 19 February 2016. During this period, 67P was at a heliocentric distance of $\sim 2.4$ AU from the Sun (Fig. 1i, red); Rosetta moved in a hyperbolic arc between $\sim 34.8$ and $34.3 \mathrm{~km}$ from 67P (Fig. 1i, blue) with a relative speed of $\sim 0.2 \mathrm{~m} \mathrm{~s}^{-1}$. Rosetta was above the southern (summer) hemisphere of 67P. The sub-spacecraft latitude changed from $-32.2^{\circ}$ to $-24.1^{\circ}$ during this interval (Fig. $1 \mathrm{~h}$, blue).

Figure 1a shows the neutral gas number density $n_{\mathrm{n}}$ at the spacecraft position measured by ROSINA/COPS, corrected for neutral composition as discussed in Galand et al. (2016). The $n_{\mathrm{n}}$ exhibited a slight decrease from $\sim 0.84 \times 10^{8} \mathrm{~cm}^{-3}$ at 0946 UT to $\sim 0.78 \times 10^{8} \mathrm{~cm}^{-3}$ at $0950 \mathrm{UT}$. Although this decrease is less than the typical $n_{\mathrm{n}}$ fluctuations due to the nucleus rotation $\left(\sim 10^{8} \mathrm{~cm}^{-3}\right.$ on 19 February), this pre-outburst decrease is also present in the plasma density (Fig. 1c) and the spacecraft potential (Fig. 1d). Following the decrease, $n_{\mathrm{n}}$ rapidly increased at a rate of $\sim 23.2 \times$ $10^{5} \mathrm{~cm}^{-3} \mathrm{~min}^{-1}$ to a peak value of $\sim 1.42 \times 10^{8} \mathrm{~cm}^{-3}$ at $1021 \mathrm{UT}$. The neutral density at Rosetta increased by a factor of $\sim 1.8$ during this outburst. Following the peak density, $n_{\mathrm{n}}$ decreased gradually at a rate of $\sim 4.2 \times 10^{5} \mathrm{~cm}^{-3} \mathrm{~min}^{-1}$, which was significantly slower than the increase rate. We note that $n_{\mathrm{n}}$ decreased to a value $\left(\sim 0.64 \times 10^{8} \mathrm{~cm}^{-3}\right.$ at $\left.1408 \mathrm{UT}\right)$ that was lower than the pre-outburst level $\left(\sim 0.84 \times 10^{8} \mathrm{~cm}^{-3}\right.$ at $\left.0946 \mathrm{UT}\right)$ before increasing again as a result of the nucleus rotation effect. It should be noted that the ROSINA/COPS neutral density measurement may have been affected by local dust increase (see Fig. 17 of Grün et al. 2016) between 1100 and 1200 UT, resulting in a possible overestimation of the neutral density.

Variations in the cometary plasma density are monitored by RPC-MIP and RPC-LAP instruments. Figure $1 \mathrm{~b}$ shows the mutual impedance spectrogram from which the plasma frequency is extracted to estimate the electron density $n_{\mathrm{e}}$ (Fig. 1c). Figure $1 \mathrm{~d}$ shows the Langmuir probe potential with respect to the floating spacecraft potential, used as an estimate of the (negative) spacecraft potential (P1 and P2 for LAP1 and LAP2, respectively). The possible difference with the total spacecraft potential is ignored here (see Odelstad et al. 2015, for more details). The parameters show similar variations as $n_{\mathrm{n}}$, i.e., the pre-outburst decrease at $\sim 0938$ UT, the abrupt increase during outburst, and the gradual decrease to or below pre-outburst level at $\sim 1400$ UT. One exception is that RPC measurements revealed a different plasma behavior from $\sim 1200$ UT onward. This is not observed in the ROSINA/COPS neutral gas measurements.

The RPC-MIP was operated in the short Debye length (SDL) mode most of the time, except for a short time interval after 1400 UT when RPC-MIP was operated in the long Debye length (LDL) mode (Fig. 1b). The Debye length varied between $\sim 50 \mathrm{~cm}$ and $\sim 100 \mathrm{~cm}$ during the outburst shown in Fig. 1. Details about the SDL and LDL modes can be found in Trotignon et al. (2007). The plasma density $n_{\mathrm{e}}$ is extracted from the RPC-MIP SDL operational mode only because the plasma frequency was above the frequency window used in the LDL operational mode. The value of $n_{\mathrm{e}}$ increased from $\sim 320 \mathrm{~cm}^{-3}$ at 0945 UT to the peak value of $\sim 1225 \mathrm{~cm}^{-3}$ at $1027 \mathrm{UT}$ (Fig. 1c). The increase in $n_{\mathrm{e}}$ was at a rate of $\sim 21.6 \mathrm{~cm}^{-3} \mathrm{~min}^{-1}$, a factor of $\sim 3.8$, much larger than the observed $\sim 1.8$ times increase in cometary neutral density (Fig. 1a). Afterwards, $n_{\mathrm{e}}$ gradually decreased at a rate of $\sim 9.0 \mathrm{~cm}^{-3} \mathrm{~min}^{-1}$ to $\sim 378 \mathrm{~cm}^{-3}$ at $1201 \mathrm{UT}$. A secondary enhancement in $n_{\mathrm{e}}$ to $\sim 874 \mathrm{~cm}^{-3}$ is noted at $1207 \mathrm{UT}$, followed by another decrease at a slower rate of $\sim 4.9 \mathrm{~cm}^{-3} \mathrm{~min}^{-1}$ until $\sim 1400 \mathrm{UT}$. The interval between $\sim 1200$ and 1400 UT is characterized by larger fluctuations in $n_{\mathrm{e}}$ compared to the interval $\sim 1000-1200 \mathrm{UT}$.

In Fig. 2 we plot RPC-MIP $n_{\mathrm{e}}$ measurements along the spacecraft trajectory around the outburst interval. The values of $n_{\mathrm{e}}$ are shown by a color bar at the top. This figure clearly shows the localized nature (in space and time) of the plasma response 

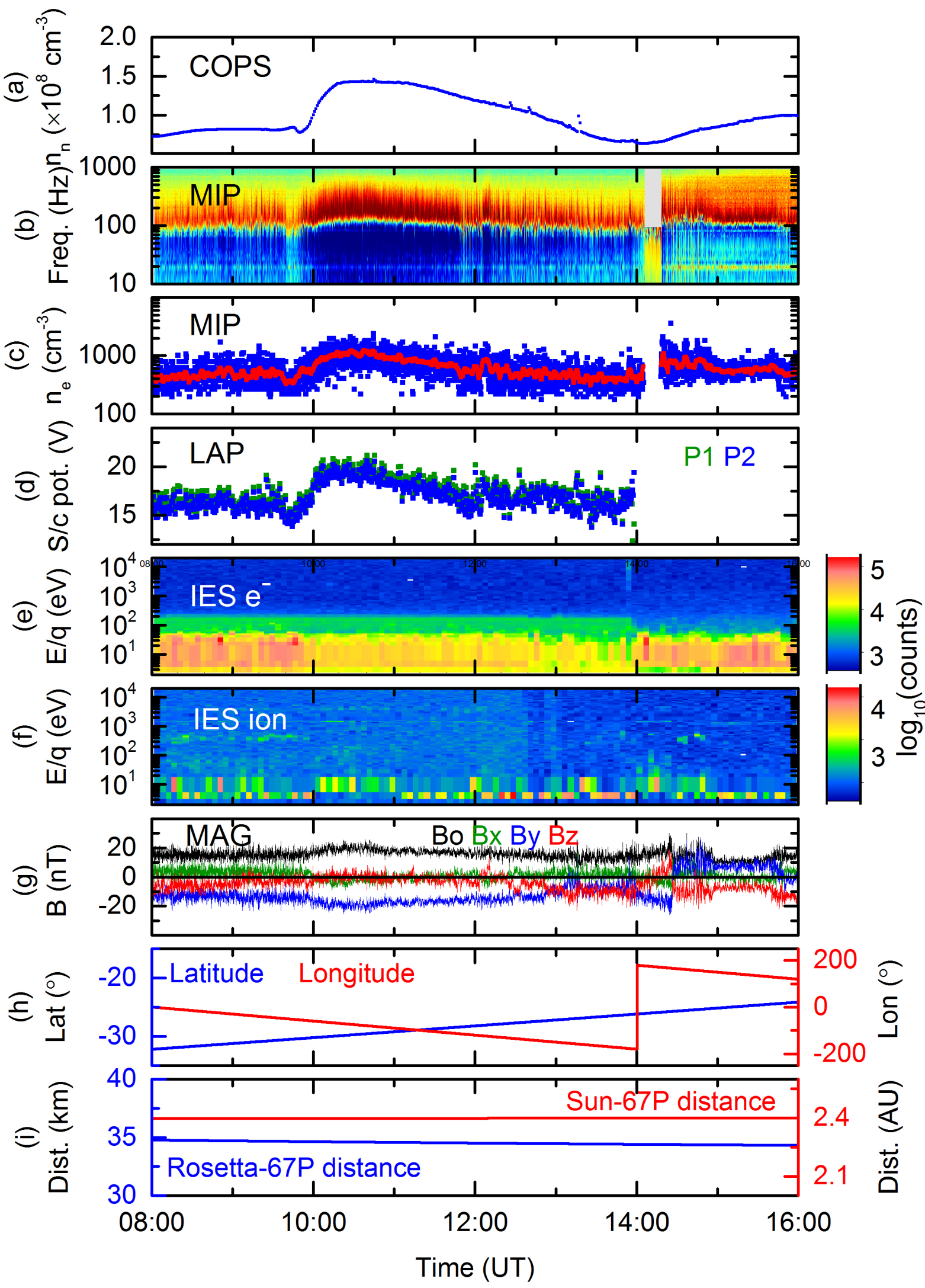

Fig. 1. RPC measurements during the outburst on 19 February 2016. From top to bottom, the panels show: a) neutral gas density from ROSINA/COPS; $b$ ) RPC-MIP plasma frequency spectrogram; $c$ ) electron density estimate from RPC-MIP (blue) along with running average (red); $d$ ) negative spacecraft potential from RPC-LAP probes 1 and 2 (P1 and P2); $e$ ) electron energy spectrogram from RPC-IES; $f$ ) ion energy spectrogram from RPC-IES; $g$ ) magnetic field components Bx, By, Bz and magnitude Bo in the cometocentric solar equatorial (CSEQ) coordinates from RPC-MAG; $h$ ) cometary latitude (blue, scale on left) and longitude (red, scale on right) of Rosetta; $i$ ) distances of the comet from the Sun (red, scale on right) and from Rosetta (blue, scale on left), respectively. 
MIP plasma density (cm-3)

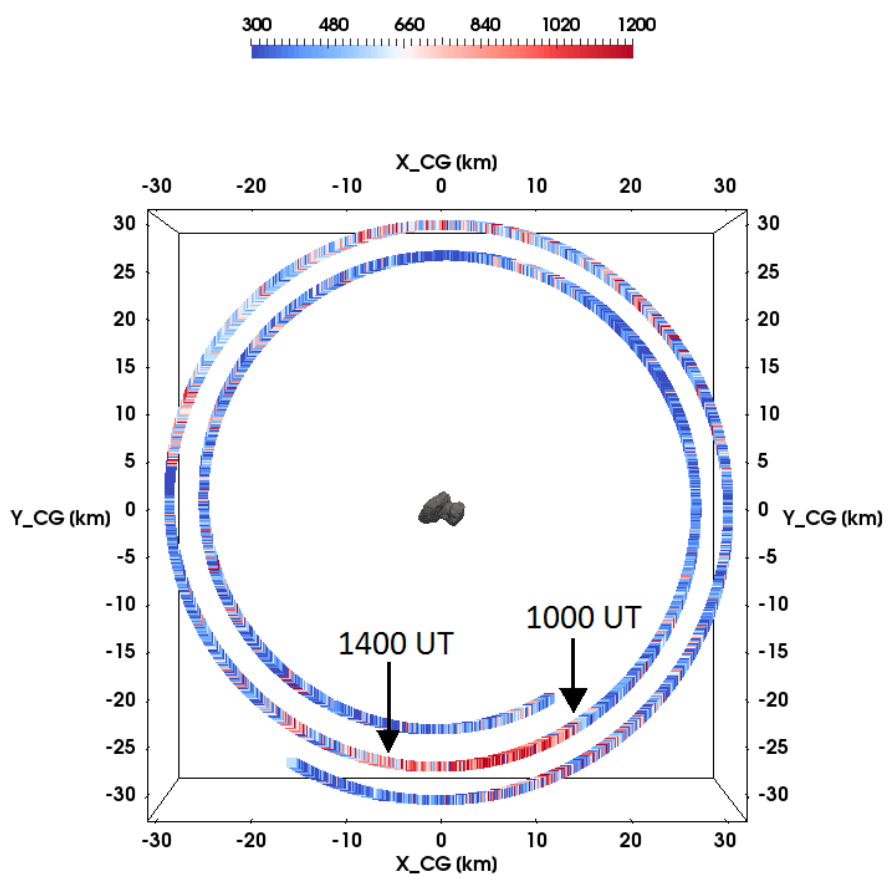

Fig. 2. Electron density estimated by RPC-MIP along the spacecraft trajectory in the reference frame of the comet on 19 February 2016.

to the cometary outburst. Lower plasma densities can be noted in the spacecraft passes $\sim 12 \mathrm{~h}$ before and after the outburst interval.

As a response to the cometary outburst, the spacecraft potential estimated by RPC-LAP increased from $\sim 14.3 \mathrm{~V}$ at $0944 \mathrm{UT}$ to $\sim 20.6 \mathrm{~V}$ at $1016 \mathrm{UT}$ (Fig. 1d). The factor of $\sim 1.4$ increase in spacecraft potential is consistent with the observed local plasma density increase of a factor of $\sim 3.8$ (Fig. 1c) for isothermal electrons. The increase is followed by a decrease at a rate of $\sim 5.4 \times 10^{-2} \mathrm{~V} \mathrm{~min}^{-1}$ to a potential of $\sim 15.2 \mathrm{~V}$ at $1155 \mathrm{UT}$, a secondary increase to $\sim 18.3 \mathrm{~V}$ at $1208 \mathrm{UT}$, and another slower decrease at a rate of $\sim 3.6 \times 10^{-2} \mathrm{~V} \mathrm{~min}^{-1}$ until $\sim 1400 \mathrm{UT}$. Unfortunately, RPC-LAP spacecraft potential observations are not available after $\sim 1400 \mathrm{UT}$.

The temperature $T_{\mathrm{e}}$ of the core electrons was estimated from the charge current balance criteria between the thermal flux of surrounding plasma electrons to the negatively charged spacecraft (not shown). For this estimation, we used the density measured by RPC-MIP, spacecraft potential measured by RPC-LAP, and the photoemission current obtained by scaling the RPC-LAP photoemission current density to the sunlit surface area of the spacecraft (Odelstad et al. 2015). The estimated $T_{\mathrm{e}}$, while noisy, was found to be $\sim 6 \pm 1 \mathrm{eV}$, with no significant change above the noise level before, during, or after the outburst.

Figures 1e and f show the energy spectrograms of electrons and ions, respectively, obtained from RPC-IES. The electron and ion fluxes are presented by uncalibrated count rates integrated over $256 \mathrm{~s}$. The values are shown by the color bars on the right. Detailed studies on the cometary ions can be found in Goldstein et al. (2015). Superthermal electron distributions near the 67P are studied by Broiles et al. (2016) and Madanian et al. (2016). The electron spectrum exhibits a decrease in the fluxes of energetic $(\sim 4-70 \mathrm{eV})$ electrons at the onset of the outburst at $\sim 1000$ UT (Fig. 1e). A further decrease is observed around 1230 UT, continuing until $\sim 1400$ UT. In the response of the

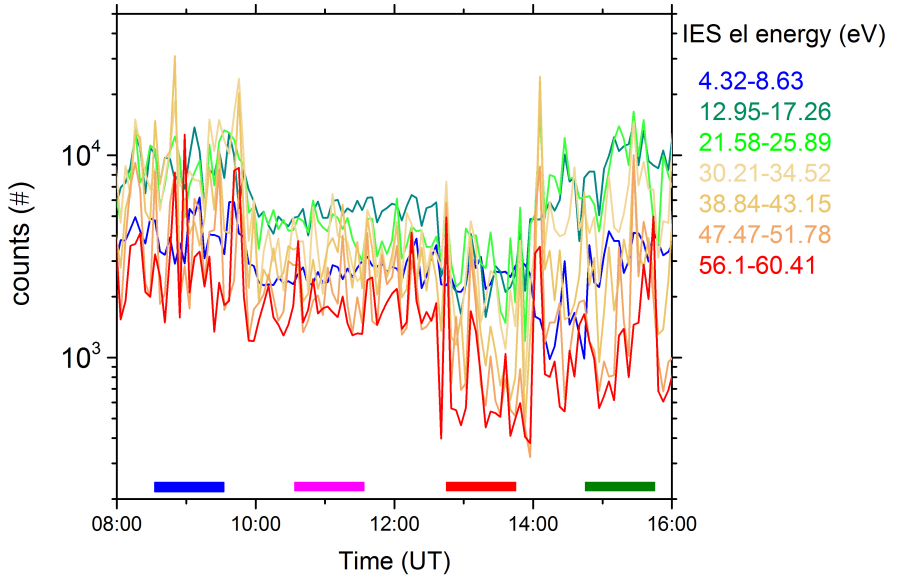

Fig. 3. Energetic electron fluxes estimated by RPC-IES. The energy levels of the electrons are shown at the right. The horizontal bars show $1 \mathrm{~h}$ time intervals. See text for details.

outburst, solar wind ions $\left(\mathrm{H}^{+}\right.$ions with energy in the range $\sim 400-600 \mathrm{eV}$ ) are observed to disappear, at least from the RPCIES field of view, after $\sim 1000$ UT (Fig. 1f). The reappearance of the solar wind is observed during 1430-1500 UT. Cometary ion fluxes $(<10 \mathrm{eV})$ are enhanced, due to the increased negative spacecraft potential (Fig. 1d). There is also evidence of accelerated cometary ions up to energies of a few hundred $\mathrm{eV}$. The electron responses are discussed in greater detail in Sect. 2.2.

It should be mentioned that the RPC-ICA was turned on at 0400 UT in high time resolution mode, which is $2 \mathrm{D}$ and does not cover solar wind energies. It was turned off again at 1200 UT. The fluxes of ions with energy above $\sim 60 \mathrm{eV}$ were found to decrease after the onset of the outburst (not shown).

The magnetic field measured by RPC-MAG exhibits interesting behavior during the outburst (Fig. 1g). The Bx, By, and $\mathrm{Bz}$ components of the magnetic field in the cometocentric solar equatorial (CSEQ) coordinate system are shown by green, blue, and red curves, respectively, along with the magnetic field magnitude Bo in black. Prominent features are the increase in Bo and the rotation in the field direction. Detailed analyses of the magnetic field indicate the disappearance of low-frequency waves (tens of $\mathrm{mHz}$ ), usually observed in the close plasma environment of the comet (Richter et al. 2015, 2016; Koenders et al. 2016; Meier et al. 2016), at the time of the outburst (see Sects. 2.3 and 2.4).

\subsection{Suprathermal electron population behavior during the outburst}

In Fig. 1e a decrease in the energetic electron fluxes is observed during the outburst, while the total electron density increases (Fig. 1c) and the electron thermal temperature is observed to remain constant during the same period (see Sect. 2.1). To quantify the energetic electron flux decrease, the variation of the electron flux (count rates) in the energy range $\sim 4-70 \mathrm{eV}$ is plotted (Fig. 3). The flux decrease starts at $\sim 0945$ UT, with a secondary decrease at $\sim 1230$ UT continuing until $\sim 1400$ UT. The decrease in the electron flux depends on the energy level. The electron flux with energy in the range of $12.95-17.26 \mathrm{eV}$ exhibits a factor of $\sim 3$ decrease from $\sim 13.8 \times 10^{3}$ at 0937 UT to $\sim 4.3 \times 10^{3}$ at 1011 UT. A further flux decrease is noted from a value of $\sim 5.9 \times 10^{3}$ at 1236 UT to a value of $\sim 1.6 \times 10^{3}$ at $1257 \mathrm{UT}$, a factor of $\sim 4$ decrease. In the energy range of $30.21-34.52 \mathrm{eV}$, the electron flux decreased from $\sim 20.4 \times 10^{3}$ at 0945 UT to a 


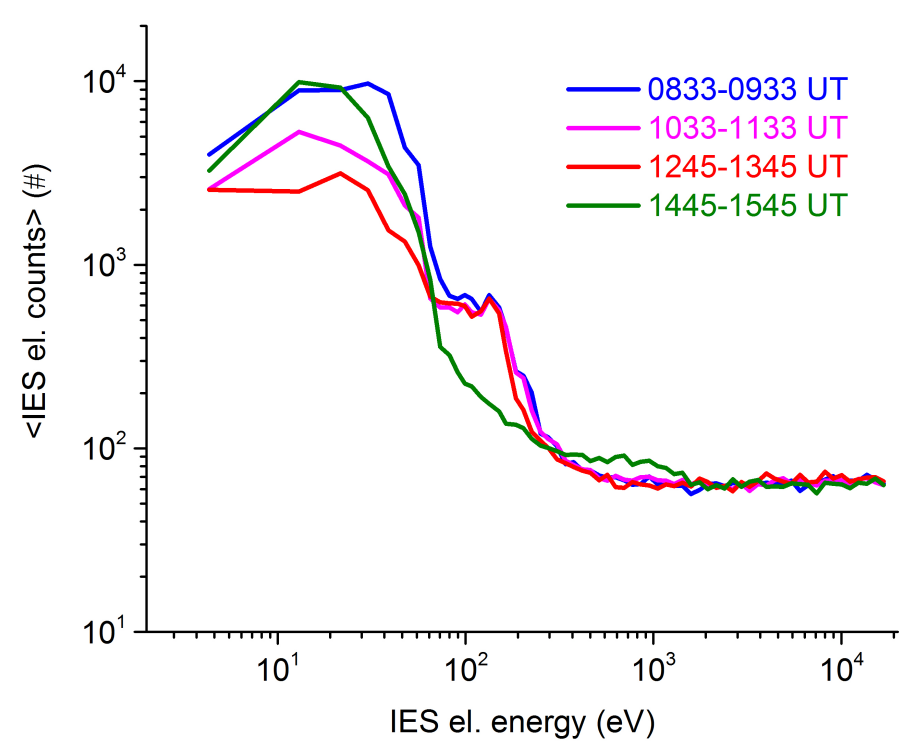

Fig. 4. Average uncalibrated electron counts (RPC-IES) as a function of energy at the time periods indicated. The noise level is about 80 counts, reached above $200 \mathrm{eV}$.

value of $\sim 2.4 \times 10^{3}$ at 1002 UT. The electron flux further decreased from a value of $\sim 2.8 \times 10^{3}$ at 1240 UT to $\sim 1.2 \times 10^{3}$ at 1253 UT. Thus, electrons flux in the 30.21-34.52 eV energy bin decreased by factors of $\sim 9$ and $\sim 2$ at $\sim 1002$ UT and $\sim 1253$ UT, respectively.

We chose four $1 \mathrm{~h}$ time intervals from Fig. 3: 0833-0933 UT (before the outburst), 1033-1133 UT (first step electron flux decrease between 1000 and 1200 UT during the outburst), 12451345 UT (second step electron flux decrease between 1200 and 1400 UT during the outburst), and 1445-1545 UT (after the outburst). These are shown by color bars at the bottom of Fig. 3 . The electron counts integrated over azimuth and elevation angles during the four intervals are estimated separately and plotted as a function of electron energy in Fig. 4. For energies in the range $\sim 10-80 \mathrm{eV}$, significant lower fluxes are noted during the outburst compared to the fluxes before and after the events. It should be noted that the noise level is $\sim 80$ above $200 \mathrm{eV}$.

\subsection{Magnetic field rotation during the cometary outburst}

The RPC-MAG measurements of the magnetic field are shown in the top four panels of Fig. 5. They are repeated from Fig. 1g for detailed analyses. The $1 \mathrm{~h}$ average of Bo is superposed over the high-resolution data (1 s). On average, Bo increased from $\sim 14.8 \mathrm{nT}$ at $0940 \mathrm{UT}$ to $\sim 19.8 \mathrm{nT}$ at $1025 \mathrm{UT}$. It decreased slowly at the rate of $\sim 3.6 \times 10^{-2} \mathrm{nT} \mathrm{min}^{-1}$ to the value of $\sim 13.0 \mathrm{nT}$ at $1337 \mathrm{UT}$.

An interesting feature of the magnetic field is its rotation during the outburst. We estimated the angle of rotation of the magnetic field vector with respect to the magnetic field vector averaged over 0800-0900 UT interval, well before the outburst. The variation in the rotation at every $10 \mathrm{~min}$ interval is shown in the bottom panel of Fig. 5. The onset of the outburst is marked by a sharp increase in the rotation angle from $\sim 13.9^{\circ}$ at 0950 UT to $\sim 36.4^{\circ}$ at $1010 \mathrm{UT}$, after which the rotation decreased slowly. Another sharp increase in rotation is noted around 1200 UT, which corresponds to a local extremum in plasma density and spacecraft potential (Fig. 1). At $\sim 1430$ UT, the By and Bz components abruptly changed their polarities from negative to positive and from positive to negative, respectively. This results in

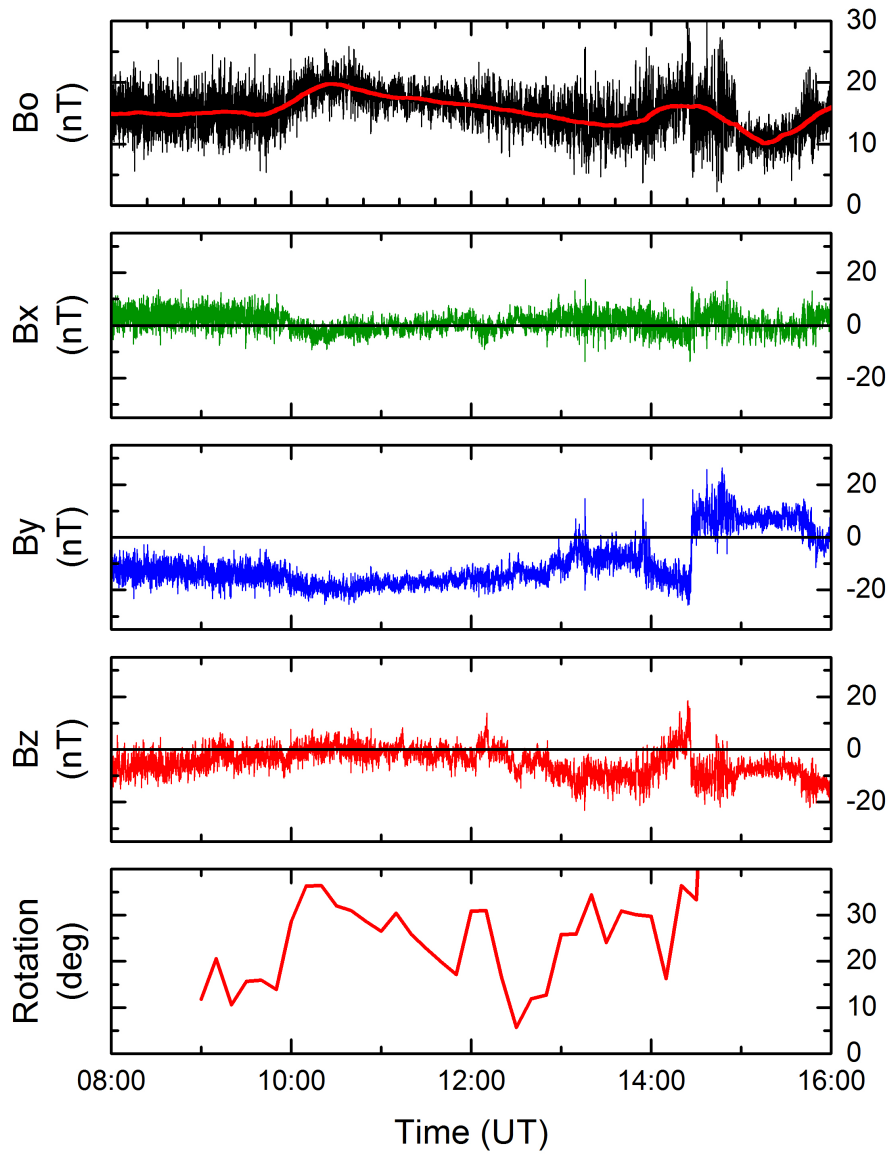

Fig. 5. Magnetic field measured by RPC-MAG. From top to bottom: magnetic field magnitude $\mathrm{Bo}, \mathrm{Bx}, \mathrm{By}$, and $\mathrm{Bz}$ components in CSEQ coordinates, and rotation of the field. The red curve in the Bo panel shows the $1 \mathrm{~h}$ average of the high-resolution Bo (1 s).

a sharp increase in rotation angle from $\sim 33.3^{\circ}$ at 1430 UT to $\sim 110.7^{\circ}$ at $1450 \mathrm{UT}$ (not shown). This appears to mark the end of the cometary outburst influence on the magnetic field.

The observed simultaneous amplitude increase and rotation in the magnetic field is consistent with a local pile-up and draping of magnetic field lines around the denser cometary plasma cloud generated during the outburst, acting as a local induced magnetosphere. The magnetic field rotation (Fig. 1g) observed around 1000 UT occurs exactly during the neutral density and plasma responses to the outburst (Figs. 1a and c, respectively), and evolves on the same timescale. To make it even clearer, in Fig. 6 we show both responses: the variations of the cometary plasma density and the magnetic field orientation and amplitude along the spacecraft trajectory in the $Y Z$ (left panel) and the $X Z$ (right panel) planes in the CSEQ reference frame. On the other hand, the magnetic field rotation observed later, around 1430 UT, together with a very local plasma density increase (not seen in the neutral density, as expected for such a current sheet) is more typical of a solar wind magnetic field rotation, similar to the current sheets reported in Volwerk et al. (2017).

\subsection{Wave characteristics during the cometary outburst}

The RPC-MAG data is used to construct the field variances in order to study the wave characteristics during the cometary outburst. The $10 \mathrm{~s}, 30 \mathrm{~s}, 1 \mathrm{~min}$, and 3 min variances were calculated from magnetic field data at $1 \mathrm{~s}$ time resolution and then were 
Table 1. Average normalized variances of $\mathrm{Bx}, \mathrm{By}$, and $\mathrm{Bz}$ during four $1 \mathrm{~h}$ intervals.

\begin{tabular}{lcccc}
\hline \hline Interval & $10 \mathrm{~s}\left(\times 10^{-3}\right)$ & $30 \mathrm{~s}\left(\times 10^{-3}\right)$ & $1 \min \left(\times 10^{-3}\right)$ & $3 \min \left(\times 10^{-3}\right)$ \\
\hline Before outburst 0833-0933 UT & $17.0,13.2,11.3$ & $25.7,21.8,18.6$ & $29.1,24.6,21.6$ & $32.3,27.2,26.5$ \\
During outburst 1033-1133 UT & $3.4,2.7,5.0$ & $6.7,4.9,9.5$ & $8.4,6.1,11.9$ & $11.1,7.6,14.6$ \\
During outburst 1245-1345 UT & $17.0,14.2,12.9$ & $30.0,24.8,22.4$ & $37.0,35.7,29.2$ & $42.2,40.9,34.4$ \\
After outburst 1445-1545 UT & $15.1,17.9,12.2$ & $28.3,27.9,24.1$ & $43.4,37.5,27.9$ & $52.8,44.6,36.0$ \\
\hline
\end{tabular}

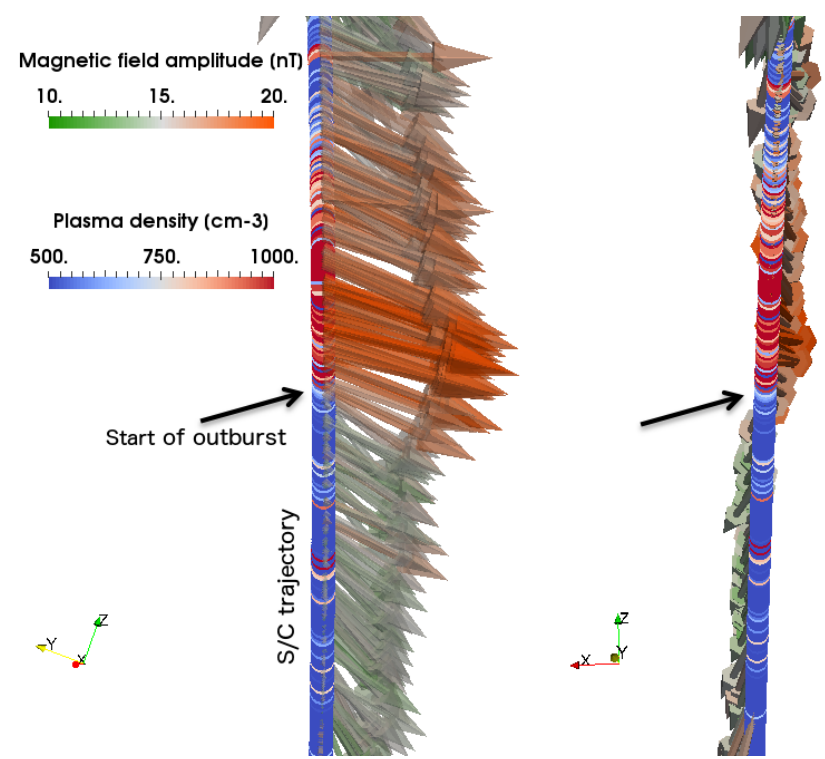

Fig. 6. Plasma density and magnetic field variations along the Rosetta spacecraft trajectory projected in the $Y Z$ (left panel) and XZ (right panel) planes in the CSEQ frame. The spacecraft trajectory is directed from bottom to top, with the beginning of the outburst indicated by a black arrow. The plasma density is color-coded along the trajectory. Vectors indicate the magnetic field direction; length and color are both coded with the magnetic field amplitude.

used to make $3 \mathrm{~min}$ averages of the quantities. The variances give the amount of wave power for frequencies up to the variance value (Tsurutani et al. 1982; Hajra et al. 2013). For example, the $3 \mathrm{~min}$ average of the $10 \mathrm{~s}$ variances represent the average wave power occurring in the $1000 \mathrm{mHz}$ (corresponding to the highest $1 \mathrm{~s}$ resolution of the data used) to $100 \mathrm{mHz}$ wave frequency range. The $30 \mathrm{~s}$ variances give the wave power occurring in the $1000 \mathrm{mHz}$ to $33.3 \mathrm{mHz}$ wave power range. If the $10 \mathrm{~s}$ variance is subtracted from the $30 \mathrm{~s}$ variance, the resultant value is the amount of wave power which was present for wave frequencies between $100 \mathrm{mHz}$ and $33.3 \mathrm{mHz}$. The variances can be used to determine an average wave power and a low-resolution power spectrum. We normalized the variances by dividing them by the square of the magnetic field magnitude.

The left panels of Fig. 7 show the 3 min averages of the $10 \mathrm{~s}, 30 \mathrm{~s}, 1 \mathrm{~min}$, and $3 \mathrm{~min}$ normalized variances of the $\mathrm{Bx}$, $\mathrm{By}$, and $\mathrm{Bz}$ components of the magnetic field. The average normalized variances of $\mathrm{Bx}, \mathrm{By}$, and $\mathrm{Bz}$ during the four $1 \mathrm{~h}$ intervals mentioned in Sect. 2.2 (Fig. 3) are listed in Table 1. A large variation can be noted in the variances in the periods inside and outside the outburst from Table 1 and Fig. 7 (left panels). The normalized variances are significantly smaller during 10331133 UT around the peak of the outburst compared to those before and after the outburst. This signifies wave attenuation during peak phase of the outburst. Enhancement in the variances during
1245-1345 UT interval is consistent with the large fluctuations in $n_{\mathrm{e}}$ variation during the 1200-1400 UT period (Fig. 1c).

The middle panels of Fig. 7 show the Morlet wavelet spectrum of the magnetic field components. The spectrum indicates the temporal variation of the dominant frequencies in the magnetic field (Torrence \& Compo 1998). The wavelet reconstructed magnetic field components in the $10-100 \mathrm{mHz}$ frequency range are shown in the right panels of Fig. 7. Small yellow and green regions within the frequency range of $\sim 10$ $100 \mathrm{mHz}$ in the frequency spectrum indicate magnetic "singing comet waves" (Richter et al. 2015, 2016; Koenders et al. 2016; Meier et al. 2016). These singing comet waves disappear or become weakened between $\sim 1000$ and 1300 UT, as shown by the reduced wave amplitudes shown in the right panels. This is most prominent in the By component (in the ecliptic perpendicular to the Sun-67P line).

Figure 8 shows the magnetic energy in the wave, estimated as $E_{B} \sim \delta B_{\mathrm{o}}^{2} / 2 \mu_{\mathrm{o}}$, where $\delta B_{\mathrm{o}}$ is the magnetic field amplitude in the $10-100 \mathrm{mHz}$ range. The energy $E_{B}$ suffers attenuation during 1000-1300 UT compared to the periods before and after the outburst. The mean (median) values of $E_{B}$ during the intervals before (0800-0900 UT), during (1000-1300 UT), and after (1330-1500 UT) the outburst are $\sim 1.2 \times 10^{-11} \mathrm{~J}, 0.6 \times 10^{-11} \mathrm{~J}$, and $1.4 \times 10^{-11} \mathrm{~J}$, respectively $\left(8.4 \times 10^{-12} \mathrm{~J}, 4.1 \times 10^{-12} \mathrm{~J}\right.$ and $9.9 \times 10^{-12} \mathrm{~J}$, respectively). The magnetic energy associated with these waves decreased by a factor of $\sim 2$ during the outburst.

\section{Discussion}

\subsection{Ionization balance during the cometary outburst}

We make a comparison between the cometary plasma density response to the outburst and an ionospheric model in order to identify the main sources of ionization and to assess the main processes involved during the cometary outburst. According to Galand et al. (2016), photoionization by the solar EUV and electron-impact ionization are the main sources of the cometary plasma. For increased neutral densities, evidence of partial energy degradation of the electron energy tail and cooling of the full electron population are shown. Based on the balance between the ionization rate and radial transport, the cometary plasma density $n_{\mathrm{i}}$ at a cometocentric distance $r$ is expected to vary as (see Galand et al. 2016)

$n_{\mathrm{i}}(r)=\frac{\left(v^{h v}+v^{e-}\left(r_{0}\right)\right)\left(r-r_{\mathrm{s}}\right)}{u_{\mathrm{i}}(r)} n_{\mathrm{n}}(r)$,

where $v^{h v}$ and $v^{e-}$ are respectively the solar EUV photoionization and electron-impact ionization frequencies, $u_{\mathrm{i}}$ is the ion bulk velocity, $n_{\mathrm{n}}$ is the neutral gas density, $r_{0}$ is the cometocentric distance of Rosetta, and $r_{\mathrm{s}}$ is the cometocentric distance of the comet surface. Galand et al. (2016) showed that at large heliocentric distances, as is the case here, the chemistry loss timescale for electron-ion dissociative recombination is significantly longer than the transport timescale, and therefore dissociative recombination is a negligible process. The neutral gas 

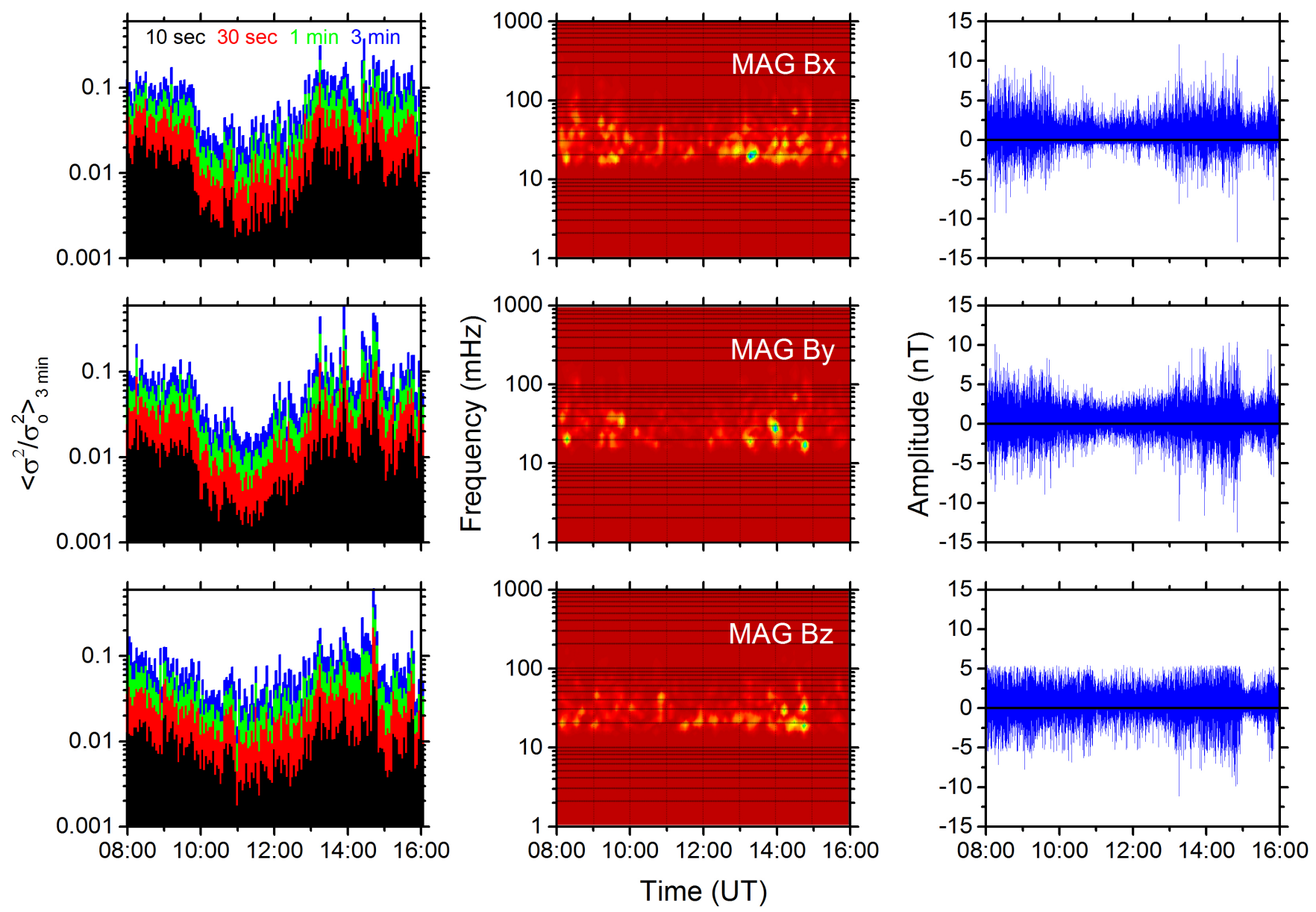

Fig. 7. Left panels: nested normalized variances of the magnetic field components Bx, By, and Bz for $10 \mathrm{~s}$ (black), $30 \mathrm{~s}$ (red), 1 min (green), and $3 \mathrm{~min}$ (blue) intervals. Middle panels: Morlet wavelet spectrum. Right panels: wavelet reconstructed magnetic fields in the $10-100 \mathrm{mHz}$ range. From top to bottom: Bx, By, and Bz components.

density $n_{\mathrm{n}}$ from ROSINA/COPS is shown in Fig. 9 (top, solid line) along with the sub-spacecraft latitude (dashed line). The $n_{\mathrm{n}}$ has not been corrected for neutral composition. The neutral composition correction for ROSINA/COPS $n_{\mathrm{n}}$ based on the ROSINA-Double Focusing Mass Spectrometer (DFMS) is included in the ionization frequencies instead (see Galand et al. 2016).

The photoionization frequency $v^{h v}$ and the electron-impact ionization frequency $v^{e-}$ are plotted in blue and with red circles, respectively, in Fig. 9 (bottom). Each is the sum of the individual frequencies associated with a neutral species and weighted by the volume mixing ratio of that species derived from observations by ROSINA-DFMS (Galand et al. 2016). The photoionization frequency $v^{h v}$ of the neutral species $\left(\mathrm{H}_{2} \mathrm{O}, \mathrm{CO}_{2}\right.$, or $\left.\mathrm{CO}\right)$ is derived from the photoionization cross sections of the neutral species and the solar flux measurements from the Thermosphere Ionosphere Mesosphere Energetics and Dynamics (TIMED)Solar EUV Experiment (SEE) at the Earth (Woods et al. 2005). We used the daily solar flux on 18 February 2016 to take into account the Earth-Sun-comet 67P angle. The flux was also extrapolated in heliocentric distance from the Earth to $2.4 \mathrm{AU}$, the position of 67P on 19 February. From 0600 UT the neutral composition has ratios with respect to $\mathrm{H}_{2} \mathrm{O}$ of 0.33 for $\mathrm{CO}_{2}$ and of 0.1 for CO. From 0900 UT these ratios slowly decrease, and after 1015 UT, the composition is mostly $\mathrm{H}_{2} \mathrm{O}$. This change in composition is solely responsible for the change of $7 \%$ seen in the photoionization frequency. The frequency $v^{e-}$ is derived from the electron-impact ionization cross sections of the neutral species considered $\left(\mathrm{H}_{2} \mathrm{O}, \mathrm{CO}_{2}, \mathrm{CO}\right)$ and the RPC-IES electron flux density. The latter results from the integration of the electron intensity over elevation and azimuthal angles and assuming isotropy for blind spots (Clark et al. 2015). The electron energy associated with the flux density was also corrected for the spacecraft potential. A full description of the calculation of the electronimpact ionization frequency is given in Galand et al. (2016). The MCP efficiency and in-flight calibration were taken into account. The frequency $v^{e-}$ is decreasing by a factor of 3 at the start of the outburst, consistent with Fig. 1e. It partially recovers after 1400 UT. In addition, while $v^{e-}$ represents $60 \%$ of $v^{h v}$ before the outburst, it drops to almost $20 \%$ during the outburst.

A comparison of the modeled ionospheric densities with the RPC-MIP electron density is shown in Fig. 10. The modeled electron densities are derived from Eq. (1), which results from the balance between photoionization and transport. The blue (red) area corresponds to simulations assuming photoionization alone (both photoionization and electron-impact ionization); each colored area expands from a lower boundary corresponding to an ion outflow velocity $u_{\mathrm{i}}$ of $900 \mathrm{~m} \mathrm{~s}^{-1}$ to an upper boundary corresponding to $u_{\mathrm{i}}$ of $570 \mathrm{~m} \mathrm{~s}^{-1}$. This range of values is driven by the derived value of $620 \mathrm{~m} \mathrm{~s}^{-1}$ for the neutral outflow velocity observed by the Microwave Instrument on the Rosetta Orbiter (MIRO; Gulkis et al. 2007) on 19 February (Grün et al. 2016) and associated with an uncertainty of $\pm 50 \mathrm{~m} \mathrm{~s}^{-1}$ (Biver 2016, pers. comm.). Before the outburst, photoionization is the 


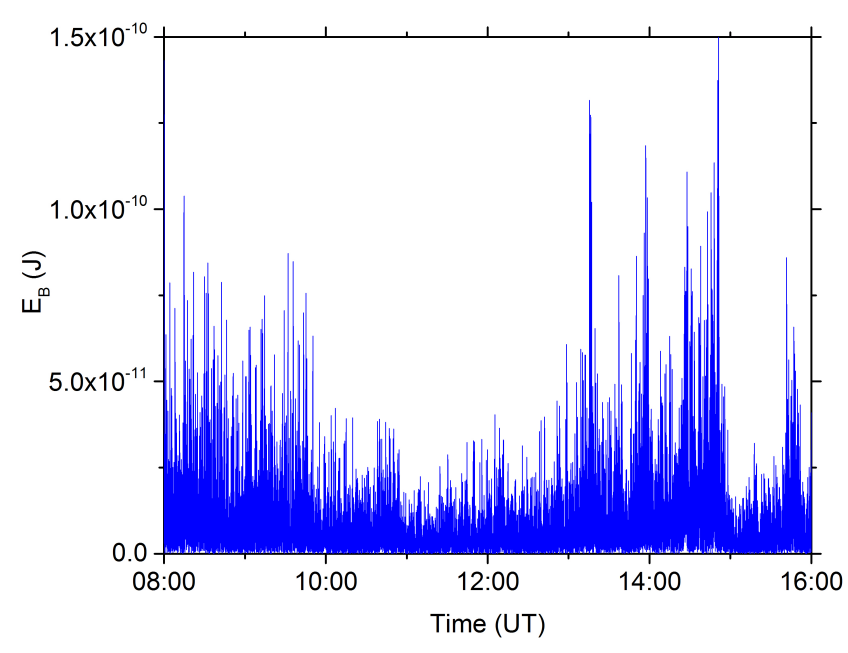

Fig. 8. Magnetic energy $\left(E_{B}\right)$ in the $10-100 \mathrm{mHz}$ frequency range during the outburst.

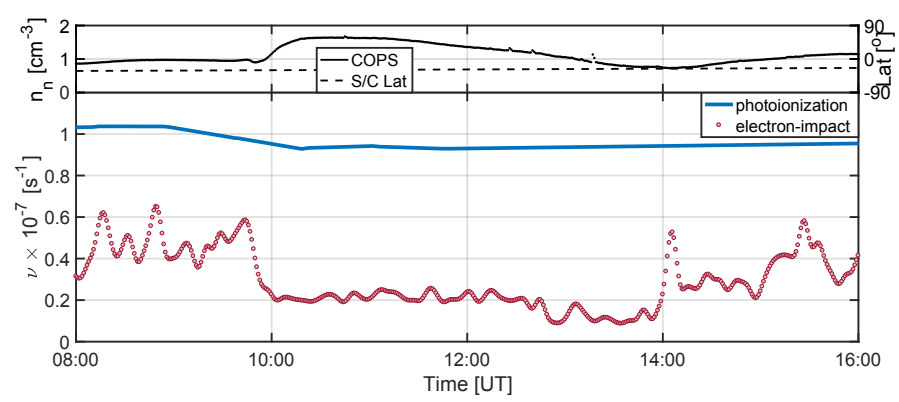

Fig. 9. Top: ROSINA/COPS neutral density $n_{\mathrm{n}}$ not corrected for neutral composition (solid line) and sub-spacecraft latitude (dashed line). Bottom: photoionization (blue solid line) and electron-impact ionization (red circles) frequencies, derived from TIMED-SEE solar flux at the Earth and extrapolated to the comet 67P and from RPC-IES electron intensities, respectively (see Galand et al. 2016).

prime source of ionization. The contribution of electron-impact ionization is modest and an ion outflow velocity of $900 \mathrm{~m} \mathrm{~s}^{-1}$ (lower red boundary in $n_{\mathrm{i}}$ ) is required to explain the observations. Just before the outburst (near 0945 UT), a combination of decreased neutral density $(8 \%)$ (with a still high $u_{\mathrm{i}}>900 \mathrm{~m} \mathrm{~s}^{-1}$ ) drives a decrease in the electron density. At the peak of the outburst between 1000 UT and 1100 UT, photoionization alone cannot explain the observed electron densities if we consider a realistic range of values for the ion bulk velocity. Electron-impact ionization combined with the ion outflow velocity similar to the neutral velocity $\left(570 \mathrm{~m} \mathrm{~s}^{-1}\right)$ is required to explain the RPC-MIP observations. Later, around 1300 UT, the neutral density returns to its pre-outburst values (Fig. 9, top panel) and the modeled ionization densities agree with the observations when considering pre-outburst high $u_{\mathrm{i}}$ values on the order of $800-900 \mathrm{~m} \mathrm{~s}^{-1}$. Between 1400 UT and 1500 UT, additional processes not included in the model, such as small-scale plasma dynamics, take place and increase the electron density and its variability. Finally, although electron-impact ionization frequency represents less than $40 \%$ of the total ionization, features seen in $v^{e-}$ but not in the other input parameters also seem to be observed in the RPCMIP measurements $\left(n_{\mathrm{e}}\right)$, such as the peak near $1400 \mathrm{UT}$ and the drop near 1545 UT (Fig. 1c).

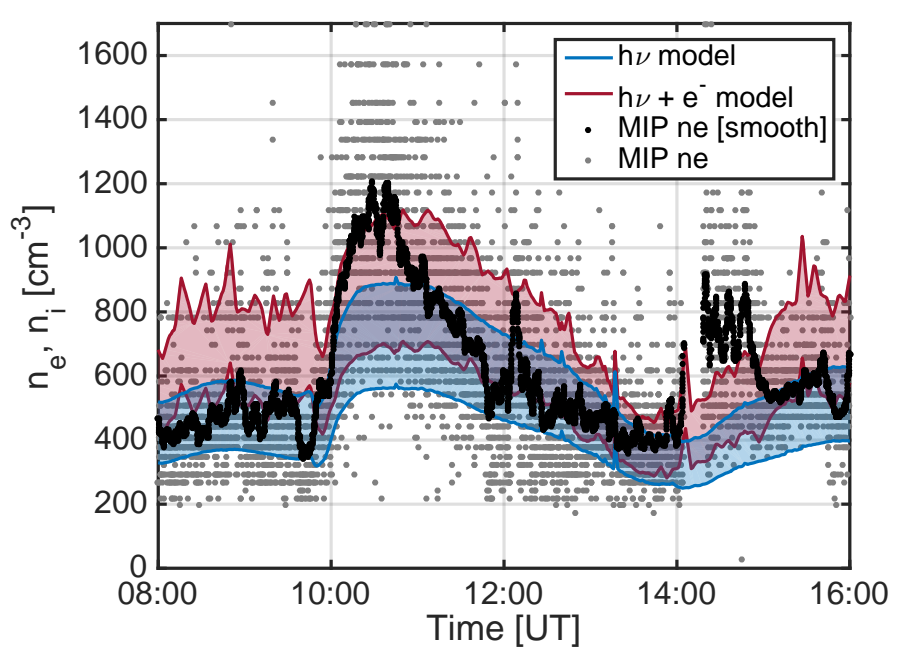

Fig. 10. Electron density from RPC-MIP $n_{\mathrm{e}}$ plotted in high-resolution (gray dots) and smoothed over 60 points (black dots) on 19 February 2016. The modeled ionospheric densities derived assuming photoionization alone and both photoionization and electron-impact ionization are shown in blue and red, respectively. The colored shaded areas represent the range of ion bulk velocities considered from $900 \mathrm{~m} \mathrm{~s}^{-1}$ (lower boundary in $n_{\mathrm{i}}$ ) to $570 \mathrm{~m} \mathrm{~s}^{-1}$ (upper boundary in $n_{\mathrm{i}}$ ).

\subsection{Disappearance of the singing comet waves}

One interesting result is the disappearance or weakening of $\sim 10$ $100 \mathrm{mHz}$ waves in the local magnetic field during the peak of the outburst (Fig. 7). The low-frequency $(\sim 40 \mathrm{mHz})$ "singing comet waves" at 67P were first detected in August 2014 when Rosetta arrived at 67P (Richter et al. 2015). More recently, Richter et al. (2016) reported detection of singing comet waves from August 2014 until March 2015, when the heliocentric distance of 67P from the Sun varied from 3.6 to 2.0 AU. The waves are shown to be quasi-harmonic, large-amplitude $(\delta B / B \sim 1)$, low-frequency, and compressional in nature. They are quite different from the large-amplitude waves and turbulence previously observed at the comets 21P/Giacobini-Zinner (Tsurutani \& Smith 1986), 1P/Halley (Glassmeier et al. 1989), and 26P/Grigg-Skjellerup (Glassmeier \& Neubauer 1993).

A modified ion-Weibel instability (Chang et al. 1990) associated with newborn cometary ion current under low cometary activity was proposed as a possible source mechanism for this new type of waves at 67P (Meier et al. 2016). Accordingly, under the low cometary activity conditions, when $67 \mathrm{P}$ was at $\sim 2.0 \mathrm{AU}$ from the Sun or beyond, newborn ions moving transversely to the ambient magnetic field and the solar wind flowing in the direction of the electric field constitute a cross-field current that can trigger the ion-Weibel instability. The instability was shown to be associated with a frequency of $\sim 40 \mathrm{mHz}$ for the $\mathrm{H}_{2} \mathrm{O}$ ion mode. During the present outburst, RPC-IES shows a disappearance of solar wind ions $(\sim 400-600 \mathrm{eV})$, at least from its field of view, possibly owing to a strong solar wind deflection (Fig. 1f). The solar wind ions are the key ingredients necessary to produce the cross-field electric field (and a cross-field ion current). The absence of solar wind ions may weaken the cross-field instability required for the low-frequency wave generation and explain why the comet stops singing. Further studies will be required to understand the influence of neutral and plasma density increases on the wave instability, if any. 


\section{Summary and conclusion}

The paper presents, for the first time, a detailed analysis of the impact of a cometary brightness outburst on the local characteristics of the cometary induced magnetosphere-ionosphere system. This is done by in situ combined measurements of neutral gas, plasma, and magnetic field by Rosetta as the spacecraft was orbiting the comet 67P. The ROSINA/COPS and RPC observations during the 67P outburst taking place on 19 February 2016 can be summarized as follows:

1. The onset of the outburst was preceded by pre-outburst decreases in neutral gas density at the spacecraft position (ROSINA/COPS), local electron density (RPC-MIP), and negative spacecraft potential (RPC-LAP) at $\sim 0950$ UT (Fig. 1). The relative timing of these decreases are not understood yet and may require further studies.

2. With the onset of the outburst at $\sim 1000 \mathrm{UT}$, the neutral gas density $n_{\mathrm{n}}$ increased by a factor of $\sim 1.8$, which is significantly larger than the $n_{\mathrm{n}}$ fluctuations due to comet nucleus rotational effects during this time period. Following the outburst peak, $n_{\mathrm{n}}$ decreased at a slower rate $\left(\sim 4.2 \times 10^{5} \mathrm{~cm}^{-3} \mathrm{~min}^{-1}\right)$ than the rate of increase $(\sim 23.2 \times$ $10^{5} \mathrm{~cm}^{-3} \mathrm{~min}^{-1}$ ) before the peak (Fig. 1a). Measurements may have been affected by the dust event during decreasing phase.

3. The local plasma (electron) density $n_{\mathrm{e}}$ increased by a factor of $\sim 3.8$ at a rate of $\sim 21.6 \mathrm{~cm}^{-3} \mathrm{~min}^{-1}$ as a response to the outburst. The $n_{\mathrm{e}}$ peak at 1027 UT was followed by a two-step decrease, at a rate of $\sim 9.0 \mathrm{~cm}^{-3} \mathrm{~min}^{-1}$ until $\sim 1200 \mathrm{UT}$ and at a rate of $\sim 4.9 \mathrm{~cm}^{-3} \mathrm{~min}^{-1}$ until $\sim 1400$ UT. The $\sim 1200$ 1400 UT interval was characterized by large fluctuations in $n_{\mathrm{e}}$ (Fig. 1c).

4. The spacecraft potential (negative) increased at a rate of $\sim 19.5 \times 10^{-2} \mathrm{~V} \mathrm{~min}^{-1}$ by a factor of $\sim 1.4$, representing a local plasma density increase of a factor of $\sim 3.8$. The potential reached a peak at 1016 UT, followed by two-step decreases at rates of $\sim 5.4 \times 10^{-2} \mathrm{~V} \mathrm{~min}^{-1}$ and $\sim 3.6 \times 10^{-2} \mathrm{~V} \mathrm{~min}{ }^{-1}$ (Fig. 1d).

5. The energetic $(\sim 4-70 \mathrm{eV})$ electron fluxes exhibited a twostep decrease by factors ranging from $\sim 2$ to 9 , depending on the energy of the electrons during the outburst (Figs. 1e and 3 ).

6. Comparison between the RPC-MIP electron density and an ionospheric model based on ROSINA/COPS and RPCIES measurements shows that the increase in neutral density in the first phase of the outburst (1100-1200 UT) is not great enough to explain the maximum electron density reached during this period. The model indicates that the ion outflow velocity decreases near the outburst peak to 570$600 \mathrm{~m} \mathrm{~s}^{-1}$, values consistent with the observed neutral velocity (Grün et al. 2016). In addition, electron-impact ionization frequency, though not dominating (20-60\%), needs to be taken into account to explain the observations. The changes in neutral composition have a minor effect on the ionospheric density (Figs. 9 and 10).

7. The solar wind ions (with energy $\sim 400-600 \mathrm{eV}$ ) disappeared, at least from the field of view of the RPC-IES, between 1000 and 1400 UT during the outburst (Fig. 1f).

8. During the outburst, the local magnetic field magnitude increased by $\sim 5 \mathrm{nT}$. The field components exhibited rotation, the rotation angle being $\sim 36.4^{\circ}$ at 1010 UT (Figs. 1g, 5, and 6).
9. During the peak phase of the outburst, from $\sim 1000$ to 1200 UT, $\sim 10-100 \mathrm{mHz}$ waves (known as the singing comet waves) disappeared or diminished strongly (Fig. 7, Table 1).

In this work, we presented the first quantitative analyses of multiinstrumental, in situ observations of a cometary ionosphere (induced magnetosphere) under the influence of a cometary brightness outburst. The Rosetta spacecraft escorting the comet 67P provides only single-point measurements along the spacecraft trajectory. This reveals plasma characteristics that are localized in space and in time. On the contrary, both ground-based and spacecraft-based remote-sensing observations give access to more global measurements, integrated along lines of sight. They therefore provide a broader picture, though with lower resolution. Remote-sensing observations of comet 67P outbursts from Rosetta have shown that outburst jets are very localized in the vicinity of the nucleus (Vincent et al. 2016). The outburst studied in the present work is unique in the sense that the spacecraft trajectory happened to cross the outburst jet, therefore enabling detailed in situ measurements. If the plasma instruments from the RPC were able to thoroughly monitor changes in the cometary ionosphere during this outburst, it is worth noticing that the impact on the cometary ionosphere of other outburst jets, which were not crossed by the Rosetta spacecraft, were hardly noticed in the RPC measurements, at least up to now. This suggests that the impact of cometary outbursts on the cometary ionosphere is very local, at least in the close environment of the comet nucleus. In this regard, in situ observations contrast with remote observations of cometary brightness outburst, which rather indicate that the duration of outbursts is typically much longer than is reported here. This could be explained by the fact that ground-based observations are biased toward much larger outbursts, easier to observe remotely. It could also be an artifact associated with line of sight measurements that spatially integrate the signature of the outburst as it propagates away from the comet. Indeed, a filament or a shell would appear as a short-lived structure from in situ measurements, but as a long-lived structure from ground-based measurements. Finally, one of the surprising observations is the changes in plasma and magnetic fields that occurred prior to the neutral signature. This could be associated with the geometry of the outburst jet itself, as the spacecraft trajectory crosses it. We are confident that this first report of the impact of a cometary outburst on an induced cometary magnetosphere from in situ single-point observations will enable us to better constrain remote large-scale observations of cometary outbursts. We encourage further modeling studies in order to predict the present in situ observations, and link them to remote observations through cometary outburst simulations.

Acknowledgements. Rosetta is a European Space Agency (ESA) mission with contributions from its member states and National Aeronautics and Space Administration (NASA). The work at LPC2E/CNRS was supported by CNES, ESEP, and by ANR under the financial agreement ANR-15-CE31-0009-01. Work at Imperial College London is supported by STFC of UK under grants ST/K001051/1 and ST/N000692/1. This work has made use of AMDA and RPC quick-look database to provide an initial overview of the event studied. This is provided through a collaboration between the Centre de Données e la Physique des Plasmas (CDPP) (supported by CNRS, CNES, Observatoire de Paris, and Université Paul-Sabatier, Toulouse) and Imperial College London (supported by the UK Science and Technology Facilities Council). Portions of the research were conducted at the Jet Propulsion Laboratory, California Institute of Technology, under contract with NASA. The data used in this paper will soon be made available on the ESA planetary science archive and is available upon request until that time. We wish to thank T. E. Cravens for helpful and constructive comments that helped improve our paper greatly. 


\section{References}

Balsiger, H., Altwegg, K. Bochsler, P., Eberhardt, P., et al. 2007, Space Sci. Rev. 128,745

Beech, M., \& Gauer, K. 2002, Earth Moon Planets, 88, 211

Belton, M. J. S., Thomas, P., Carcich, B., et al. 2013, Icarus, 222, 477

Broiles, T. W., Livadiotis, G., Burch, J. L., et al. 2016, J. Geophys. Res., 121, 7407

Burch, J. L., Goldstein, R., Cravens, T. E., et al. 2007, Space Sci. Rev., 128, 697

Carr, C., Cupido, E., Lee, C. G. Y., et al. 2007, Space Sci. Rev., 128, 629

Chang, C. L., Wong, H. K., \& Wu, C. S. 1990, Phys. Rev. Lett., 65, 1104

Clark, G., Broiles, T. W., Burch, J. L., et al. 2015, A\&A, 583, A24

Coates, A. J., \& Jones, G. H. 2009, Planet. Space Phys., 57, 1175

Eddington, A. S. 1910, Roy. Astron. Soc., LXX. 5, 442

Eriksson, A. I., Boström, R., Gill, R., et al. 2007, Space Sci. Rev., 128, 729

Filonenko, V. S., \& Churyumov, K. I. 2006, Adv. Space Res., 38, 1940

Galand, M., Héritier, K. L., Odelstad, E., et al. 2016, MNRAS, 462, S331

Glassmeier, K. H. 2017, Phil. Trans. R. Soc. A, 375, 20160256

Glassmeier, K. H., Coates, A. J., Acuna, M. H., et al. 1989, J. Geophys. Res., 94, 37

Glassmeier, K. H., \& Neubauer, F. M. 1993, J. Geophys. Res., 98, 20921

Glassmeier, K. H., Boehnhardt, H., Koschny, D., Kührt, E., \& Richter, I. 2007a, Space Sci. Rev., 128, 1

Glassmeier, K. H., Richter, I., Diedrich, A., et al. 2007b, Space Sci. Rev., 128, 649

Goldstein, R., Burch, J. L., Mokashi, P., et al. 2015, Geophys. Res. Lett., 42 3093

Gronkowski, P. 2007, Astron. Nachr., 328, 126

Gronkowski, P. 2009, MNRAS, 397, 883

Gronkowski, P., \& Sacharczuk, Z. 2010, MNRAS, 408, 1207

Gronkowski, P., \& Wesolowski, M. 2015, MNRAS, 451, 3068

Grün, E., Agarwal, J., Altobelli, N., et al. 2016, MNRAS, 462, S220

Gulkis, S., Frerking, M., Crovisier, J., et al. 2007, Space Sci. Rev., 128, 561

Hajra, R., Echer, E., Tsurutani, B. T., \& Gonzalez, W. D. 2013, J. Geophys. Res., 118,5626

Huebner, W. F., \& Weigert, A. 1966, Z. Astrophys., 64, 185
Hughes, D. W. 1990, Roy. Astron. Soc., Quant. J., 31, 69 Ibadov, S. 2012, Adv. Space Res., 49, 467

Intrilligator, D. S., \& Dreyer, M. 1991, Nature, 353, 407

Keller, H. U., Küppers, M., Fornasier, S., et al. 2007, Icarus, 191, 241

Koenders, C., Perschke, C., Goetz, C., et al. 2016, A\&A, 594, A66

Madanian, H., Cravens, T. E., Rahmati, A., et al. 2016, J. Geophys. Res., 121, 5815

Meier, P., Glassmeier, K. H., \& Motschmann, U. 2016, Ann. Geophys., 34, 691

Miles, R. 2016, Icarus, 272, 356

Moreno, F., Ortiz, J. L., Santos-Sanz, P., et al. 2008, ApJ, 677, L63

Niedner, M. B. 1980, ApJ, 241, 820

Nilsson, H., Lundin, R., Lundin, K., et al. 2007, Space Sci. Rev., 128, 671

Odelstad, E., Eriksson, A. I., Edberg, N. J. T., et al. 2015, Geophys. Res. Lett., 42, 10126

Qi, C., Hogerheijde, M. R., Jewitt, D., Gurwell, M. A., \& Wilner, D. J. 2015, ApJ, 799, 110

Rettig, T. W., Tegler, S. C., Pasto, D. J., \& Mumma, M. J. 1992, ApJ, 398, 293

Richter, I., Koenders, C., Auster, H. U., et al. 2015, Ann. Geophys., 33, 1031

Richter, I., Auster, H. U., Berghofer, G., et al. 2016, Ann. Geophys., 34, 609

Sekanina, Z. 2008a, Int. Comet Quart., 30, 3

Sekanina, Z. 2008b, Int. Comet Quart., 30, 63

Szegö, K., Glassmeier, K.-H., Bingham, R., et al. 2000, Space Sci. Rev., 94, 429

Tambovtseva, L. V., \& Shestakova, L. I. 1999, Planet. Space Sci., 47, 319

Torrence, C., \& Compo, G. P. 1998, Bull. Am. Meteor. Soc., 79, 61

Trotignon, J. G., Michau, J. L., Lagoutte, D., et al. 2007, Space Sci. Rev., 128, 713

Tsurutani, B. T., \& Smith, E. J. 1986, Geophys. Res. Lett., 13, 259

Tsurutani, B. T., Smith, E. J., Anderson, R. R., et al. 1982, J. Geophys. Res., 87, 6060

Vincent, J. B., A'Hearn, M. F., Lin, Z. Y., et al. 2016, MNRAS, 462, S184

Volwerk, M., Jones, G. H., Broiles, T., et al. 2017, J. Geophys. Res., 122, 3308

West, R. M., Hainaut, O., \& Smette, A. 1991, A\&A, 246, L77

Whitney, C. 1955, ApJ, 122, 190

Woods, T. N., Eparvier, F. G., Bailey, S. M., et al. 2005, J. Geophys. Res., 110, A01312 